\title{
THE USE OF THE LINEAR FORM OF DYNAMICAL EQUATIONS OF THE SATELLITE ATTITUDE CONTROL SYSTEM FOR ITS ANALYSIS AND SYNTHESIS
}

\author{
Meirbek Moldabekov, Anna Sukhenko, Darya Shapovalova \\ AALR "Institute of Space Technique and Technology", Almaty, Kazakhstan \\ e-mail: sukhenko.a@istt.kz \\ SulEimen YelubayeV \\ Al-Farabi Kazakh National University, Almaty, Kazakhstan \\ e-mail: yelubayev.s@istt.kz
}

\begin{abstract}
At present, the methods based on using linearized dynamical equations are applied for synthesis of an attitude control system of a satellite with nonlinear dynamics. Linearized equations describe the satellite dynamics approximately, which is the main their disadvantage. This article shows that basing on the angular momentum theorem, the nonlinear dynamical equations of the satellite attitude control system can be represented in the form of linear differential equations with variable coefficients, which makes it possible to use engineering methods of stability analysis and analysis of transient quality in the process of synthesis of the satellite attitude control system.
\end{abstract}

Key words: synthesis, stability analysis, attitude control system, satellite

\section{Introduction}

As a rule, the control law of the satellite attitude control system is based on the PD-regulator (Moldabekov et al., 2015; Chaurais et al., 2013; Narkiewicz et al., 2020; Mehrjardi et al., 2014; Ran et al., 2016; Nasrolahi and Abdollahi, 2016) and the dynamics of the satellite attitude control system is described by nonlinear differential equations. The interest arises for the question of whether it is possible to represent the dynamical equations of the satellite attitude control system in the linear form. If it is possible, it enables engineers to use the rich arsenal of convenient methods of dynamical analysis and synthesis of parameters for linear dynamic systems.

At present, linearized equations of the dynamics of the satellite attitude control system are used to analyze the stability of motion and the synthesis of control systems from the condition of ensuring the required quality of transient processes of satellite orientation (Moldabekov et al. 2017; Psiaki, 2001; Galvao et al. 2016; Doruk, 2009; Rossa et al., 2013; Nasrolahi and Abdollahi, 2016, Ocampo, 2019). Expanding the functions on the right and left sides of the equations of motion in a Taylor series and discarding high-order nonlinear terms is the main method of linearizing the nonlinear equations of satellite motion, which is used by many authors (Doruk, 2009; Blanke and Larsen, 2010). Linearized equations describe the satellite dynamics approximately, which is the main their disadvantage. The question about the global stability of the original system of nonlinear equations under the condition of stability of the linearized system arises in the process of the control system analysis and synthesis. The authors have a fairly large arsenal of synthesis methods for linearized systems in the case of using linear control laws but the question of the global stability of the original system of nonlinear equations remains open. The work in solving this problem is carried out, in particular, Zhou (2015) obtained necessary and sufficient conditions for the Lyapunov stability of the linearized system of equations, but 
not the global stability. Nasrolahi and Abdollahi (2016) presented the results of studying the stability of a nonlinear system using the Lyapunov function method which provided the sufficient conditions for stability.

It is known from the stability theory that the local stability of the original nonlinear system follows from the stability of the linearized system and global stability does not follow from the stability of the linearized system (Demidovich, 1967). It is also well known that the Lyapunov function method provides sufficient conditions for the stability of a nonlinear system, but it does not provide the necessary conditions for its stability. This article shows that basing on the angular momentum theorem (Markeev, 1999; Knudsen and Hjorth, 1995), the nonlinear dynamics equations of the satellite attitude control system can be represented in the linear form. The article presents results of studying the linear form of equations, in particular, the necessary and sufficient conditions of the global asymptotic stability of the linear system that entail the global asymptotic stability of the original nonlinear system. This result is qualitatively different from the result based on the linear approximation obtained by Zhou (2015), Nasrolahi and Abdollahi (2016). In fact, it provides necessary and sufficient conditions for the global stability of the original nonlinear system. Also, a method for synthesis of parameters of the control law is proposed in this article. This method provides the required indicators of quality of transient processes based on the use of a given distribution of roots of the characteristic equation of a linear system and the scale of transition to normalized time for linear systems.

\section{Mathematical model of the satellite attitude control system}

Let us introduce the following coordinate systems and their notation:

- $O X Y Z$ is a stationary inertial coordinate system (ICS) with the origin in the center of mass of the Earth (point $O$ ). Its $O X$ axis lies in the equatorial plane, and it is directed to the spring equinox. Its $O Z$ axis coincides with the axis of Earth rotation, and it is directed to the north pole of the Earth. $O Y$ axis complements the system to the right system;

- Cxyz is a body coordinate system (BCS) with the origin in the center of mass of the satellite (point $C$ ). Its axes coincide with the main central axes of inertia of the satellite.

To describe the dynamics of rotational motion of the satellite, we apply the dynamic Euler equations for the rotational motion of a rigid body with a fixed point (Markeev, 1999)

$$
\mathbf{J} \dot{\boldsymbol{\omega}}+\boldsymbol{\omega} \times\left(\mathbf{J} \boldsymbol{\omega}+\mathbf{J}_{r} \boldsymbol{\omega}_{r}\right)=-\mathbf{J}_{r} \dot{\boldsymbol{\omega}}_{r}
$$

where $\mathbf{J}=\left\{J_{1}, J_{2}, J_{3}\right\}$ is the diagonal $(3 \times 3)$ matrix of the inertia tensor of the satellite; $J_{1}, J_{2}, J_{3}$ are the main central moments of inertia of the satellite; $\boldsymbol{\omega}=\left[\omega_{1}, \omega_{2}, \omega_{3}\right]^{\mathrm{T}}$ is the vector of the absolute angular velocity of the satellite in projections on the axis of the body coordinate system $C x y z ; \mathbf{J}_{r}=\left\{J_{r 1}, J_{r 2}, J_{r 3}\right\}$ is the diagonal $(3 \times 3)$ reaction wheel inertia tensor matrix; $J_{r 1}, J_{r 2}, J_{r 3}$ are the moments of inertia of the reaction wheels installed along the $x, y, z$ axes, respectively; $\boldsymbol{\omega}_{r}=\left[\omega_{r 1}, \omega_{r 2}, \omega_{r 3}\right]^{\mathrm{T}}$ is the vector of angular velocities of the reaction wheels.

Equation (2.1) characterizes the change of the vector of the angular velocity of the satellite under the action of the vector of control moments of the reaction wheels. In the scalar form, these equations are

$$
\begin{aligned}
& J_{1} \dot{\boldsymbol{\omega}}_{1}+\left(J_{3}-J_{2}\right) \omega_{2} \omega_{3}+J_{r 3} \omega_{r 3} \omega_{2}-J_{r 2} \omega_{r 2} \omega_{3}=M_{1} \\
& J_{2} \dot{\boldsymbol{\omega}}_{2}+\left(J_{1}-J_{3}\right) \omega_{1} \omega_{3}+J_{r 1} \omega_{r 1} \omega_{3}-J_{r 3} \omega_{r 3} \omega_{1}=M_{2} \\
& J_{3} \dot{\boldsymbol{\omega}}_{3}+\left(J_{2}-J_{1}\right) \omega_{1} \omega_{2}+J_{r 2} \omega_{r 2} \omega_{1}-J_{r 1} \omega_{r 1} \omega_{2}=M_{3}
\end{aligned}
$$

where

$$
M_{i}=-J_{r i} \dot{\omega}_{r i} \quad i=1,2,3
$$

are the control moments of the reaction wheels. 
We take the feedback control law in the form of a linear function

$$
M_{i}=-h_{i} \omega_{i}-\alpha_{i} \phi_{i} \quad i=1,2,3
$$

where $\dot{\phi}_{i}=\omega_{i}, h_{i}, \alpha_{i}$ are the unknown arbitrary parameters of the control law which must be determined from the conditions of stability of motion and providing the required characteristics of the transient process of orientation of the satellite.

\section{Representation of the dynamical equations of the satellite attitude control system in the linear form}

Let us write the system of differential equations (2.2) in the normal Cauchy form

$$
\begin{aligned}
& \dot{\phi}_{1}=\omega_{1} \\
& \dot{\boldsymbol{\omega}}_{1}=-\frac{1}{J_{1}}\left[h_{1} \omega_{1}+\alpha_{1} \phi_{1}+\left(J_{3} \omega_{3}+J_{r 3} \omega_{r 3}\right) \omega_{2}-\left(J_{2} \omega_{2}+J_{r 2} \omega_{r 2}\right) \omega_{3}\right] \\
& \dot{\phi}_{2}=\omega_{2} \\
& \dot{\boldsymbol{\omega}}_{2}=-\frac{1}{J_{2}}\left[h_{2} \omega_{2}+\alpha_{2} \phi_{2}-\left(J_{3} \omega_{3}+J_{r 3} \omega_{r 3}\right) \omega_{1}+\left(J_{1} \omega_{1}+J_{r 1} \omega_{r 1}\right) \omega_{3}\right] \\
& \dot{\phi}_{3}=\omega_{3} \\
& \dot{\boldsymbol{\omega}}_{3}=-\frac{1}{J_{3}}\left[h_{3} \omega_{3}+\alpha_{3} \phi_{3}+\left(J_{2} \omega_{2}+J_{r 2} \omega_{r 2}\right) \omega_{1}-\left(J_{1} \omega_{1}+J_{r 1} \omega_{r 1}\right) \omega_{2}\right]
\end{aligned}
$$

where $\omega_{i}(t), \omega_{r i}(t), i=1,2,3$ are continuously limited functions of time $t$ due to limited power of the reaction wheel engines. It is obvious that the system of nonlinear differential equations (3.1) has a trivial solution.

The system of nonlinear differential equations (3.1) in the matrix form can be written as

$$
\dot{\mathbf{X}}=\mathbf{A X}+\mathbf{f}(\mathbf{X})
$$

where $\mathbf{X}=\left[x_{1}, \ldots, x_{6}\right]^{\mathrm{T}} \equiv\left[\phi_{1}, \omega_{1}, \phi_{2}, \omega_{2}, \phi_{3}, \omega_{3}\right]^{\mathrm{T}}, \mathbf{f}(\mathbf{X})=\left[f_{1}(X), \ldots, f_{6}(X)\right], \mathbf{A}=\mathbf{A}_{6 \times 6}-$ quasi-diagonal matrix with constant elements

$$
\begin{aligned}
& \mathbf{A}=\left[\begin{array}{cccccc}
0 & 1 & 0 & 0 & 0 & 0 \\
-\frac{\alpha_{1}}{J_{1}} & -\frac{h_{1}}{J_{1}} & 0 & 0 & 0 & 0 \\
0 & 0 & 0 & 1 & 0 & 0 \\
0 & 0 & -\frac{\alpha_{2}}{J_{2}} & -\frac{h_{2}}{J_{2}} & 0 & 0 \\
0 & 0 & 0 & 0 & 0 & 1 \\
0 & 0 & 0 & 0 & -\frac{\alpha_{3}}{J_{3}} & -\frac{h_{3}}{J_{3}}
\end{array}\right] \\
& f_{1}(X)=0 \\
& f_{3}(X)=0 \\
& f_{2}(X)=\frac{1}{J_{1}}\left[-\left(J_{3} \omega_{3}+J_{r 3} \omega_{r 3}\right) \omega_{2}+\left(J_{2} \omega_{2}+J_{r 2} \omega_{r 2}\right) \omega_{3}\right] \\
& f_{5}(X)=0
\end{aligned}
$$

Let us introduce linear functions of the angular velocities of the satellite and reaction wheels expressing the angular moments along the three axes of rotation of the satellite

$$
J_{i} \omega_{i}+J_{r i} \omega_{r i}=C_{i} \quad i=1,2,3
$$


According to the angular momentum theorem (Markeev, 1999) in the case when the external forces and moments acting on the satellite are equal to zero, $C_{i}<\infty$ are constants in the projections onto the fixed axes of the ICS determined by the initial conditions for the angular velocities of the satellite and reaction wheels around the coordinate axes

$$
C_{i}=J_{i} \omega_{i}(0)+J_{r i} \omega_{r i}(0) \quad i=1,2,3
$$

However, in the projections on the axis of the BCS, $C_{i}<\infty$ will be functions of its angles of rotation relative to the ICS. In this case, the values $C_{i}<\infty$ tend to constants when the indicated rotation angles tend to zero.

It can be shown that if the trivial solution of the system of equations (3.1) is asymptotically stable according to Lyapunov (Demidovich, 1967), i.e. the angles of rotation of the BCS relative to the ICS tend to zero, then the angular moments along the three axes of rotation of the satellite tend to their limit values

$$
\lim _{t \rightarrow \infty} C_{i}\left[\omega_{i}(t), \omega_{r i}(t)\right]=\lim _{t \rightarrow \infty}\left[J_{i} \omega_{i}(t)+J_{r i} \omega_{r i}(t)\right]=J_{r i} \omega_{r i}^{0}=C_{i}^{0} \quad i=1,2,3
$$

Then the angular moments along the three axes of rotation of the satellite can be represented as continuous bounded functions of time $t$

$$
C_{i}(t)=C_{i}^{0}+B_{i}(t) \quad i=1,2,3 \quad t \in[0, \infty)
$$

where

$$
\lim _{t \rightarrow \infty} B_{i}(t)=0 \quad i=1,2,3
$$

Taking into account equalities (3.3) and (3.6), the functions $\mathbf{f}(\mathbf{X})$ in equations (3.2) can be written as linear functions of angular velocities $\omega_{i}, i=1,2,3$

$$
\begin{array}{ll}
f_{1}(X, t)=0 & f_{2}(X, t)=-\frac{1}{J_{1}}\left(C_{3}(t) \omega_{2}-C_{2}(t) \omega_{3}\right) \\
f_{3}(X, t)=0 & f_{4}(X, t)=-\frac{1}{J_{2}}\left(C_{1}(t) \omega_{3}-C_{3}(t) \omega_{1}\right) \\
f_{5}(X, t)=0 & f_{6}(X, t)=-\frac{1}{J_{3}}\left(C_{2}(t) \omega_{1}-C_{1}(t) \omega_{2}\right)
\end{array}
$$

Accordingly, matrix Eq. (3.2) taking into account equalities (3.6) can be written in the form

$$
\dot{\mathbf{X}}=\left[\mathbf{A}+\mathbf{C}^{0}+\mathbf{B}(t)\right] \mathbf{X}
$$

where $\mathbf{A}+\mathbf{C}^{0}+\mathbf{B}(t)$ is the matrix with constant and time-variable elements

$$
\mathbf{A}+\mathbf{C}^{0}=\left[\begin{array}{cccccc}
0 & 1 & 0 & 0 & 0 & 0 \\
-\frac{\alpha_{1}}{J_{1}} & -\frac{h_{1}}{J_{1}} & 0 & -\frac{C_{3}^{0}}{J_{1}}-\frac{B_{3}(t)}{J_{1}} & 0 & \frac{C_{2}^{0}}{J_{1}}+\frac{B_{2}(t)}{J_{1}} \\
0 & 0 & 0 & 1 & 0 & 0 \\
0 & \frac{C_{3}^{0}}{J_{2}}+\frac{B_{3}(t)}{J_{2}} & -\frac{\alpha_{2}}{J_{2}} & -\frac{h_{2}}{J_{2}} & 0 & -\frac{C_{1}^{0}}{J_{2}}-\frac{B_{1}(t)}{J_{2}} \\
0 & 0 & 0 & 0 & 0 & 1 \\
0 & -\frac{C_{2}^{0}}{J_{3}}-\frac{B_{2}(t)}{J_{3}} & 0 & \frac{C_{1}^{0}}{J_{3}}+\frac{B_{1}(t)}{J_{3}} & -\frac{\alpha_{3}}{J_{3}} & -\frac{h_{3}}{J_{3}}
\end{array}\right]
$$

Here the elements of the quasi-diagonal matrix $\mathbf{A}$ are determined by the parameters of control law (2.3) and the elements of the constant matrix $\mathbf{C}^{0}$ are determined by the initial conditions for the angular velocities of the satellite and reaction wheels around coordinate axes (3.4).

In the particular case when the initial conditions for the system of equations (3.2) and for the angular velocities of the reaction wheels are zero, all the coefficients $C_{i}<\infty$ are equal to zero according to expressions (3.3) and the linear homogeneous system of differential equations (3.9) takes the form

$$
\dot{\mathbf{X}}=[\mathbf{A}+\mathbf{B}(t)] \mathbf{X}
$$




\section{Stability conditions of a nonlinear dynamical system}

In the work of (Moldabekov et al., 2015; Nasrolahi and Abdollahi, 2016), the stability of the satellite attitude control system with a control law in form (2.3) was studied. In (Moldabekov et al., 2015), on the basis of the second Lyapunov method, sufficient conditions for the asymptotic stability of the trivial solution of the system of nonlinear equations of dynamics (3.2) were obtained, and the region of asymptotic stability in the parameter space of the control law was found. Let us show that for the asymptotic stability of nonlinear system (3.2) it is necessary and sufficient that a homogeneous linear system of differential equations with constant coefficients is asymptotically stable

$$
\dot{\mathbf{X}}=\left[\mathbf{A}+\mathbf{C}^{0}\right] \mathbf{X}
$$

Indeed, according to Theorem 2 ch. 2 p. 12 in the work of (Demidovich, 1967), if the system with constant matrix (4.1) is asymptotically stable and conditions (3.7) are satisfied, then the perturbed system with variable matrix (3.9) is also asymptotically stable. Hence, due to the equivalence of the perturbed linear system of differential equations (3.9) and the nonlinear system of equations (3.2), it follows that if the system with constant matrix (4.1) is asymptotically stable and conditions (3.7) are satisfied, then nonlinear system (3.2) is also asymptotically stable.

Conversely, from expressions (3.5) and (3.6) it follows that for satisfying conditions (3.7), it is necessary that the nonlinear system of differential equations (3.2) be asymptotically stable.

Thus, the following statement is proved: for asymptotical stability of the nonlinear system of differential equations (3.2) it is necessary and sufficient that the system with constant matrix (4.1) be asymptotically stable.

Corollary 1. If nonlinear system (3.2) is asymptotically stable, then it is globally asymptotically stable since, according to (Demidovich, 1967), if a linear system with constant matrix (4.1) is asymptotically stable, then it is also globally asymptotically stable.

Corollary 2. The area of global asymptotic stability of nonlinear system (3.2) in the space of parameters of control law (2.3) can be found by the conditions of asymptotic stability of the linear system with constant coefficients (4.1).

However, the use of system (4.1) for engineering calculations when analyzing the stability is not very convenient, since the constant elements $C_{i}^{0}$ of the matrix of system (3.9) take different, beforehand unknown values depending on the initial angular velocity of the satellite and reaction wheels.

In this connection, a question arises whether it is possible to use the following truncated homogeneous linear system of differential equations for the stability analysis and synthesis of parameters according to the given quality of transients of the satellite attitude control system

$$
\dot{\mathbf{X}}=\mathbf{A X}
$$

in which the constant elements of matrix $\mathbf{A}$ are determined only by the parameters of control law (2.3) and are independent of the initial conditions for the angular velocities of the satellite and reaction wheels.

Let us show that with a certain choice of parameters of control law (2.3) based on the asymptotic stability conditions and required transient quality parameters of the satellite attitude control system, its asymptotic stability can be ensured under any initial conditions with respect to the angular velocities of rotation of the satellite and reaction wheels, i.e. its global asymptotic stability. 
As is known from the work of (Ocampo, 2019; Blanke and Larsen, 2010; Zhou, 2015; Besekersky and Popov, 1972) the stability of a satellite attitude control system described by a system of linear differential equations with constant coefficients (4.1) is determined by location of the roots of its characteristic equation

$$
\operatorname{det}\left[\left(\mathbf{A}+\mathbf{C}^{0}\right)-\lambda \mathbf{E}\right]=0
$$

In the particular case when the initial angular velocities of the satellite and the reaction wheels are zero, the stability of the satellite orientation system is determined by the location of the roots of the characteristic equation of the truncated system of linear differential equations (4.2)

$$
\operatorname{det}(\mathbf{A}-\lambda \mathbf{E})=0
$$

With account of the fact that the matrix $\mathbf{A}$ of system (4.2) is quasi-diagonal, its characteristic polynomial can be represented as

$$
\operatorname{det}(\mathbf{A}-\lambda \mathbf{E})=\prod_{i=1}^{3}\left(\lambda^{2}+\frac{h_{i}}{J_{i}} \lambda+\frac{\alpha_{i}}{J_{i}}\right)=\sum_{i=0}^{6} a_{i} \lambda_{i}
$$

where

$$
\begin{aligned}
& a_{0}=\frac{\alpha_{1} \alpha_{2} \alpha_{3}}{J_{1} J_{2} J_{3}} \quad a_{1}=\frac{\alpha_{1} \alpha_{2} h_{3}}{J_{1} J_{2} J_{3}}+\frac{h_{1} \alpha_{2} \alpha_{3}}{J_{1} J_{2} J_{3}}+\frac{\alpha_{1} h_{2} \alpha_{3}}{J_{1} J_{2} J_{3}} \\
& a_{2}=\frac{\alpha_{1} \alpha_{2}}{J_{1} J_{2}}+\frac{\alpha_{1} \alpha_{3}}{J_{1} J_{3}}+\frac{\alpha_{2} \alpha_{3}}{J_{2} J_{3}}+\frac{h_{1} \alpha_{2} h_{3}}{J_{1} J_{2} J_{3}}+\frac{\alpha_{1} h_{2} h_{3}}{J_{1} J_{2} J_{3}}+\frac{h_{1} h_{2} \alpha_{3}}{J_{1} J_{2} J_{3}} \\
& a_{3}=\frac{h_{1}}{J_{1}}\left(\frac{\alpha_{2}}{J_{2}}+\frac{\alpha_{3}}{J_{3}}\right)+\frac{h_{2}}{J_{2}}\left(\frac{\alpha_{1}}{J_{1}}+\frac{\alpha_{3}}{J_{3}}\right)+\frac{h_{3}}{J_{3}}\left(\frac{\alpha_{1}}{J_{1}}+\frac{\alpha_{2}}{J_{2}}\right)+\frac{h_{1} h_{2} h_{3}}{J_{1} J_{2} J_{3}} \\
& a_{4}=\frac{h_{1} h_{2}}{J_{1} J_{2}}+\frac{h_{1} h_{3}}{J_{1} J_{3}}+\frac{h_{2} h_{3}}{J_{2} J_{3}}+\frac{\alpha_{1}}{J_{1}}+\frac{\alpha_{2}}{J_{2}}+\frac{\alpha_{3}}{J_{3}} \\
& a_{5}=\frac{h_{1}}{J_{1}}+\frac{h_{2}}{J_{2}}+\frac{h_{3}}{J_{3}} \quad a_{6}=1
\end{aligned}
$$

The characteristic polynomial for the matrix $\mathbf{A}+\mathbf{C}^{0}$ of the system of linear differential equations (4.1) has the form

$$
\operatorname{det}\left[\left(\mathbf{A}+\mathbf{C}^{0}\right)-\lambda \mathbf{E}\right]=\sum_{i=0}^{6} b_{i} \lambda^{i}
$$

where

$$
\begin{aligned}
& b_{0}=a_{0} \quad b_{1}=a_{1} \quad b_{2}=a_{2}+\frac{1}{J_{1} J_{2} J_{3}}\left(C_{1}^{2} \alpha_{1}+C_{2}^{2} \alpha_{2}+C_{3}^{2} \alpha_{3}\right) \\
& b_{3}=a_{3}+\frac{1}{J_{1} J_{2} J_{3}}\left(C_{1}^{2} h_{1}+C_{2}^{2} h_{2}+C_{3}^{2} h_{3}\right) \\
& b_{4}=a_{4}+\frac{C_{1}^{2}}{J_{2} J_{3}}+\frac{C_{2}^{2}}{J_{1} J_{3}}+\frac{C_{3}^{2}}{J_{1} J_{3}} \quad b_{5}=a_{5} \quad b_{6}=a_{6}
\end{aligned}
$$

In the general case, when the initial conditions with respect to the angular velocities of the satellite and the reaction wheels are nonzero, the stability of the satellite attitude control system is determined by the distribution of the roots of characteristic equation (4.3) of system (4.1). Four of the seven coefficients of the equation coincide with the coefficients of characteristic equation (4.4), these are $b_{0}, b_{1}, b_{5}, b_{6}$. The other three coefficients have additional terms depending on the values of the angular moments $C_{1}, C_{2}, C_{3}$, which satisfy the following conditions

$$
-C_{m} \leqslant C_{i} \leqslant C_{m} \quad i=1,2,3
$$


where $C_{m}=$ const $>0$ are the largest possible values of the absolute values of expressions (3.3), which in the case of zeroing the angular velocities of the reaction wheels before the satellite maneuver are defined as

$$
C_{m}=\max _{i} \max _{\omega_{0}^{i}}\left|J_{i} \omega_{0}^{i}\right|
$$

where $\omega_{0}^{i}$ is the initial angular velocity of rotation of the satellite around the corresponding coordinate axis.

Here arises the question of how the values $C_{1}, C_{2}, C_{3}$ determined by nonzero initial conditions for the angular velocity of the satellite and the rotation angles of the moving BCS relative to the ICS affect the location of the roots of characteristic equation (4.3), i.e. on stability and transient performance of the satellite attitude control system.

The exact answer to this question can be obtained by finding the roots of characteristic equation (4.3) for possible values $C_{1}, C_{2}, C_{3}$ in interval (4.7). However, its assessment using only coefficients $b_{0}-b_{6}$ of the characteristic equation is more convenient for preliminary stability analysis (Sokolov, 1972, Sokolov and Lipatov, 1970).

Dimensionless coefficients are introduced, which are called the stability indicators

$$
u_{i}=\frac{b_{i-1} b_{i+2}}{b_{i} b_{i+1}} \quad i=1, \ldots, 4
$$

A number of theorems is proved (Sokolov and Lipatov, 1970) on the stability of linear systems, from which it follows that the sufficient conditions for their stability are

$$
u_{i}<0.465
$$

Sufficient stability conditions (4.10) are satisfied for characteristic equation (4.4) with binomial coefficients corresponding to the case $C_{1}=C_{2}=C_{3}=0$

$$
u_{1}=u_{4}=0.22 \quad u_{2}=u_{3}=0.3
$$

For the boundary case when $C_{1}, C_{2}, C_{3}$ tend to infinity, it can be shown that the corresponding boundary values of the stability indicators are equal to

$$
u_{1}=u_{4}=0.333 \quad u_{2}=u_{3}=0
$$

Based on these boundary values of the stability indicators, we can assume that sufficient stability conditions (4.10) are satisfied for all intermediate values $C_{1}, C_{2}, C_{3}$ from zero to infinity. The verification of validity of this assumption is shown below by numerical examples of the location of the roots of characteristic equation (4.4) at various values of $C_{i}, i=1,2,3$, from the interval of their values from 0 to infinity.

Without loss of generality of the results, we can accept $J_{1}=J_{2}=J_{3}=J, C_{1}=C_{2}=C_{3}=C$, $h_{1}=h_{2}=h_{3}=h, \alpha_{1}=\alpha_{2}=\alpha_{3}=\alpha$. Then, for the binomial coefficients of characteristic equation (4.4), from expressions (4.5) we obtain $\alpha=J, h=2 J$. In this case, the coefficients $b$ of equation (4.6) are transformed to the form

$$
\begin{array}{llrl}
b_{0} & =1 \quad b_{1}=6 & b_{2}=15+3\left(\frac{C}{J}\right)^{2} & b_{3}=20+6\left(\frac{C}{J}\right)^{2} \\
b_{4}=15+3\left(\frac{C}{J}\right)^{2} & b_{5}=6 \quad b_{6}=1
\end{array}
$$

Let us assume $C_{m}=J$. The calculation results for 8 values of the coefficients of characteristic equation (4.3) with the increments $0.125 C_{m}$ are shown in Table 1.

The results of calculation of the corresponding values of the stability indicators are shown in Table 2.

The results of calculation of the corresponding values of the roots of characteristic equation (4.5) are shown in Table 3. 
Table 1. Coefficients of characteristic equation (4.3) depending on $C$

\begin{tabular}{|c|c|c|c|}
\hline \multirow{2}{*}{$C$} & \multicolumn{3}{|c|}{$b$} \\
\cline { 2 - 4 } & $b_{2}$ & $b_{3}$ & $b_{4}$ \\
\hline \hline $0.125 C_{m}$ & 15.05 & 20.09 & 15.05 \\
\hline $0.25 C_{m}$ & 15.18 & 20.37 & 15.18 \\
\hline $0.375 C_{m}$ & 15.42 & 20.84 & 15.42 \\
\hline $0.5 C_{m}$ & 15.75 & 21.5 & 15.75 \\
\hline $0.625 C_{m}$ & 16.17 & 22.34 & 16.17 \\
\hline $0.75 C_{m}$ & 16.68 & 23.37 & 16.68 \\
\hline $0.875 C_{m}$ & 17.29 & 24.59 & 17.29 \\
\hline$C_{m}$ & 18 & 26 & 18 \\
\hline
\end{tabular}

Table 2. Dimensionless coefficients $u$ depending on $C$

\begin{tabular}{|c|c|c|c|c|}
\hline \multirow{2}{*}{$C$} & \multicolumn{4}{|c|}{$u$} \\
\cline { 2 - 5 } & $u_{1}$ & $u_{2}$ & $u_{3}$ & $u_{4}$ \\
\hline \hline $0.125 C_{m}$ & 0.2225 & 0.2987 & 0.2987 & 0.2225 \\
\hline $0.25 C_{m}$ & 0.2236 & 0.2946 & 0.2946 & 0.2236 \\
\hline $0.375 C_{m}$ & 0.2252 & 0.2879 & 0.2879 & 0.2252 \\
\hline $0.5 C_{m}$ & 0.2275 & 0.2791 & 0.2791 & 0.2275 \\
\hline $0.625 C_{m}$ & 0.2303 & 0.2686 & 0.2686 & 0.2303 \\
\hline $0.75 C_{m}$ & 0.2335 & 0.2567 & 0.2567 & 0.2335 \\
\hline $0.875 C_{m}$ & 0.2370 & 0.2440 & 0.2440 & 0.2370 \\
\hline$C_{m}$ & 0.2407 & 0.2308 & 0.2308 & 0.2407 \\
\hline \multicolumn{4}{|r}{} \\
\hline
\end{tabular}

Table 3. Roots of characteristic equation (4.5) depending on $C$

\begin{tabular}{|c|c|c|c|c|c|c|}
\hline \multirow{2}{*}{$C$} & \multicolumn{7}{|c|}{$\lambda$} \\
\cline { 2 - 7 } & $\lambda_{1}$ & $\lambda_{2}$ & $\lambda_{3}$ & $\lambda_{4}$ & $\lambda_{5}$ & $\lambda_{6}$ \\
\hline \hline 0 & -1 & -1 & -1 & -1 & -1 & -1 \\
\hline $0.125 C_{m}$ & -1.4396 & -1.4396 & -0.9288 & -0.9288 & -0.6316 & -0.6316 \\
& $+0.4549 i$ & $-0.4549 i$ & $+0.3705 i$ & $-0.3705 i$ & $+0.1996 i$ & $-0.1996 i$ \\
\hline $0.25 C_{m}$ & -1.4074 & -1.4074 & -1.2627 & -0.7920 & -0.5653 & -0.5653 \\
& $+0.7134 i$ & $-0.7134 i$ & & & $+0.2865 i$ & $-0.2865 i$ \\
\hline $0.375 C_{m}$ & -1.5251 & -1.5251 & -1 & -1 & -0.4749 & -0.4749 \\
& $+0.9411 i$ & $-0.9411 i$ & & & $+0.2930 i$ & $-0.2930 i$ \\
\hline $0.5 C_{m}$ & -1.5910 & -1.5910 & -1 & -1 & -0.4090 & $-0.4090 i$ \\
& $+1.1657 i$ & $-1.1657 i$ & & & $+0.2997 i$ & $-0.2997 i$ \\
\hline $0.625 C_{m}$ & -1.6434 & -1.6434 & -1 & -1 & -0.3566 & -0.3566 \\
& $+1.3814 i$ & $-1.3814 i$ & & & $+0.2997 i$ & $-0.2997 i$ \\
\hline $0.75 C_{m}$ & -1.6836 & -1.6836 & -1.0802 & -0.9258 & -0.3135 & -0.3135 \\
& $+1.5927 i$ & $-1.5927 i$ & & & $+0.2965 i$ & $-0.2965 i$ \\
\hline $0.875 C_{m}$ & -1.7209 & -1.7209 & -1.0683 & -0.9361 & -0.2769 & -0.2769 \\
& $+1.8035 i$ & $-1.8035 i$ & & & $+0.2902 i$ & $-0.2902 i$ \\
\hline$C_{m}$ & -1.7541 & -1.7541 & -1 & -1 & -0.2459 & -0.2459 \\
\hline & $+2.0144 i$ & $-2.0144 i$ & & & $+0.2823 i$ & $-0.2823 i$ \\
\hline
\end{tabular}




\section{Method of synthesis of control law parameters}

It is important that the matrix $\mathbf{A}$ of truncated system (4.2) be quasi-diagonal, because it allows one to consider rotation of the satellite around three coordinate axes independently of each other. This possibility of decomposition of the dynamical problem greatly simplifies the problems of stability analysis and synthesis of parameters of the satellite attitude control system, since these problems for a 6 -th order linear differential system are reduced to the corresponding problems for three independent 2 nd order linear differential systems.

Decomposability of characteristic polynomial (4.5) of truncated system (4.2) into three similar factors means that satellite rotations around the three coordinate axes are not only independent, but also described by identical systems of two first-order differential equations. To ensure the monotony of the transient process in rotational motion of the satellite around the coordinate axes and, at the same time, to achieve the maximum speed, we set the roots of characteristic equation (4.4) real and multiple, i.e. to rotate the satellite around each of the three coordinate axes

$$
\lambda^{2}+2 \Omega_{0} \lambda+\Omega_{0}^{2}=0
$$

where $\Omega_{0}=t_{n} / t_{r}$ is the scale of the transition from the normalized transient time $t_{n}$ to real transient time $t_{r}$ (Besekersky and Popov, 1972).

The normalized time of the transient process will be determined by constructing it at $\lambda_{i}=-1$ $(i=1, \ldots, 6)$, i.e. equation (5.1) should have the form

$$
\lambda^{2}+2 \lambda+1=0
$$

Accordingly, the coefficients of characteristic equation (4.4) for the normalized time of the transient process will be binomial and equal to

$$
\begin{array}{lrrr}
a_{0}=1 & a_{1}=6 & a_{2}=15 & a_{3}=20 \\
a_{4}=15 & a_{5}=6 & a_{6}=1 &
\end{array}
$$

For a real given time of the transient process $t_{p}$, the coefficients of characteristic equation (4.4) should be equal to

$$
\begin{array}{lrrr}
a_{0}=1 & a_{1}=6 \Omega_{0} & a_{2}=15 \Omega_{0}^{2} & a_{3}=20 \Omega_{0}^{3} \\
a_{4}=15 \Omega_{0}^{4} & a_{5}=6 \Omega_{0}^{5} & a_{6}=\Omega_{0}^{6} &
\end{array}
$$

Hence, using the known expressions for the coefficients of characteristic equation (4.5), it is possible to determine the unknown parameters of the control law by solving algebraic equations for the values of the latter.

\section{Numerical studies}

For numerical simulation of transient processes on the basis of solving nonlinear equations (3.2), taking into account synthesis of the control law parameters according to the method in Section 5, we assume that the satellite moments of inertia are equal to $J_{1}=J_{2}=0.1521 \mathrm{~kg} \mathrm{~m}^{2}$, $J_{3}=0.0375 \mathrm{~kg} \mathrm{~m}^{2}$ (Chaurais et al., 2013), the initial values of the angular position are $\phi_{1}\left(t_{0}\right)=\phi_{2}\left(t_{0}\right)=\phi_{3}\left(t_{0}\right)=45^{\circ}$, the initial angular velocities of the satellite and reaction wheels are equal to zero, the transient time is set equal to the transient time $20 \mathrm{~s}$ in Chaurais et al. (2013), where the parameters of the PD controller are determined empirically. In this article, the parameters of the control law are determined according to the method in Section 5 and, 

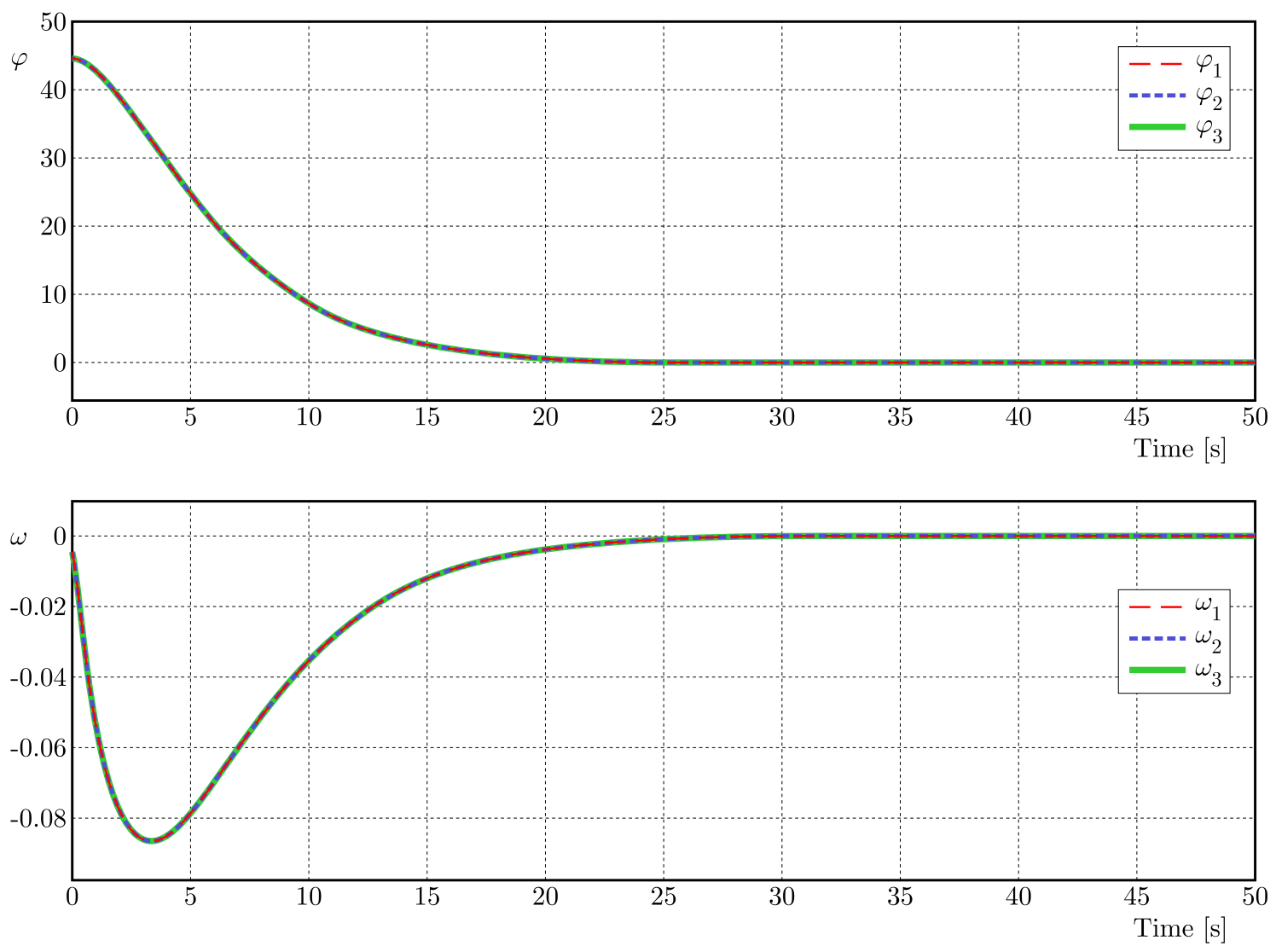

Fig. 1. Transient processes of the satellite attitude control system

with a transient time of $20 \mathrm{~s}$, are equal to $h_{1}=h_{2}=0.09126, h_{3}=0.0225, \alpha_{1}=\alpha_{2}=0.013689$, $\alpha_{3}=0.003375$. The results of numerical simulation are shown in Fig. 1.

In comparison with the simulation results given in the work of Chaurais et al. (2013), the transient processes are monotonic without oscillation, the final time of the transient process is practically the same for all three angles, which indicates high efficiency of the proposed method for synthesizing the control law parameters.

\section{Conclusions}

- It is shown that the nonlinear differential equations of the dynamics of the satellite attitude control system can be represented in a linear form, namely, in the form of linear differential equations with variable coefficients basing on the angular momentum theorem.

- Based on the study of the linear form of the original nonlinear system, necessary and sufficient conditions for its global asymptotic stability are obtained. It is proved that the region of global asymptotic stability of the original nonlinear system in the space of parameters of the control law can be found from the conditions of asymptotic stability of the linear dynamic system with constant coefficients.

- It is shown that for the analysis of stability and synthesis of parameters according to the given quality of transients of the satellite attitude control system, a truncated linear system of differential equations can be used. The constant elements of the matrix of this truncated linear system are determined only by the parameters of the control law, and these elements are independent of the initial conditions with respect to the angular velocities of the satellite and reaction wheels. 
- It is shown that with a certain choice of control law parameters, proceeding from the stability conditions and the achievement of the required parameters of the transient process of the satellite attitude control system, its stability is ensured under any initial conditions with respect to the angular velocities of the satellite rotation, i.e. the global asymptotic stability is ensured.

- It is shown that the problem of stability analysis and synthesis of parameters of the satellite attitude control system described by a system of 6 -th order linear differential equations can be reduced to corresponding problems for three independent systems of 2-nd order linear differential equations, which greatly simplifies solution of these problems.

- A method for synthesizing the parameters of the control law is proposed, which provides the required quality indicators of transient processes of satellite orientation by choosing a given distribution of the roots of the characteristic equation of the linear system and the scale of transition to the normalized time for linear systems.

The research in this article was carried out under financing of the program 008 "Applied scientific research in the field of space activities and information security".

\section{References}

1. Besekersky V.A., Popov E.P., 1972, Theory of Automatic Control Systems (in Russian), Nauka, Moscow

2. Blanke M., Larsen M.B., 2010, Satellite Dynamics and Control in a Quaternion Formulation, 2nd ed., Technical University of Denmark

3. Chaurais J.R., Ferreira H.C., Ishihara J.I., Borges R.A., Kulabukhov A.M., LARIN V.A., Belikov V.V., 2013, A high precision attitude determination and control system for the UYS-1 nanosatellite, Proceedings of 2013 IEEE Aerospace Conference, DOI: 10.1109/AERO.2013.6496981

4. Demidovich B.P., 1967, Lectures on the Mathematical Theory of Stability (in Russian), Nauka, Moscow

5. DoRuk R.Ö., 2009, Linearization in satellite attitude control with modified Rodriguez parameters, Aircraft Engineering And Aerospace Technology, 81, 3, 199-203

6. Galvao B.B., Faustino M.C.M., De Souza L.C.G., 2016, Satellite attitude control system design with nonlinear dynamics and kinematics of quaternion using reaction wheels, Proceedings of the XXXVII Iberian Latin-American Congress on Computational Methods in Engineering, DOI: https://doi.org/10.26512/ripe.v2i20.15007

7. Knudsen J.M., HJorth P.G., 1995, Elements of Newtonian Mechanics, Springer-Verlag, 1st ed.

8. Markeev A.P., 1999, Theoretical Mechanics: a Textbook for Universities (in Russian), CheRo, Moscow

9. Mehrjardi M.F., Sanusi H., Mohd. Alauddin Mohd. Ali, Taher M.A., 2014, PD Controller for three-axis satellite attitude control using discrete Kalman filter, Proceedings of 2014 International Conference on Computer, Communications and Control Technology, DOI: 10.1109/I4CT.2014.6914151

10. Moldabekov M., Akhmedov D., Yelubaev S., Alipbayev K., Sukhenko A., 2017, Optimal synthesis of satellite orientation system's parameters, Advances in the Astronautical Science, 161, 989-997

11. Moldabekov M., Yelubayev S., Alipbayev K., Sukhenko A, Bopeyev T., Mikhailenko D., 2015, Stability analysis of the microsatellite attitude control system, Applied Mechanics and Materials, 798, 297-302 
12. Narkiewicz J., Sochacki M., Zakrzewski B., 2020, Generic model of a satellite attitude control system, International Journal of Aerospace Engineering, DOI: 10.1155/2020/5352019

13. Nasrolahi S.S., AbDollahi F., 2016, Lyapunov stability analysis for non-linear satellite attitude control in the presence of states measurement error, Proceedings of 2016 4th International Conference on Control, Instrumentation and Automation, DOI: 10.1109/ICCIAutom.2016.7483137

14. Ocampo C., 2019, Modeling, Simulation, and Control of the Spacecraft Attitude Dynamics, EART University, DOI: 10.13140/RG.2.2.19952.92162

15. Psiaki M.L., 2001, Magnetic torquer attitude control via asymptotic periodic linear quadratic regulation, Journal of Guidance Control and Dynamics, DOI: 10.2514/2.4723

16. Ran D., Sheng T., Cao L., Chen X., Zhao Y., 2014, Attitude control system design and on-orbit performance analysis of nano-satellite Tian Tuo 1, Chinese Journal of Aeronautics, 27, 3, 593-601

17. Rossa F.D., Dercole F., Lovera M., 2013, Attitude stability analysis for an Earth pointing, magnetically controlled spacecraft, IFAC Proceedings Volumes, 46, 19, 518-523

18. Sokolov N.I., 1972, Lectures on the Course Theory of Automatic Regulation (in Russian), part 1, MAI, Moscow

19. Sokolov N.I., Lipatov A.V., 1970, On the application of "approximate" stability criteria for the synthesis of adaptive systems, Collection "Information materials" (in Russian), Scientific Board on Complex Problem "Cybernetics", 7, 44, Academy of Science of USSR, Moscow

20. Zhou B., 2015, On Stability of the Linearized Spacecraft Attitude Control System, arXiv:1504.00114v1 